\title{
Calculating Exceedance Probabilities Using a Distributionally Robust Method
}

\author{
Farzad Faridafshin ${ }^{\mathrm{a}}$, Bogdan Grechuk ${ }^{\mathrm{b}}$, Arvid Naess ${ }^{\mathrm{c}}$ \\ ${ }^{a}$ Sustainable Arctic Marine and Coastal Technology (SAMCoT), Centre for Research-based Innovation (CRI), \\ Norwegian University of Science and Technology, 7491 Trondheim, Norway, \\ Corresponding author, E-mail address: farzad.farid@ntnu.no ${ }^{1}$ \\ ${ }^{\mathrm{b}}$ Department of Mathematics, University of Leicester, University Road, Leicester LE1 7RH, United Kingdom, \\ E-mail address: bg83@leicester.ac.uk
}

${ }^{\mathrm{c}}$ Department of Mathematical Sciences, Norwegian University of Science and Technology, 7491 Trondheim, Norway, Email address: arvid.naess@ntnu.no

Calculation of exceedance probabilities or the inverse problem of finding the level corresponding to a given exceedance probability occurs in many practical applications. For instance, it is often of interest in offshore engineering to evaluate the wind, wave, current, and sea ice properties with annual exceedance probabilities of, e.g., $10^{-1}, 10^{-2}$, and $10^{-3}$, or socalled 10-year, 100-year, and 1000-year values. A methodology is provided in this article to calculate a tight upper bound of the exceedance probability, given any probability distribution from a wide range of commonly used distributions. The approach is based on a generalization of the Chebyshev inequality for the class of distributions with a logarithmically concave cumulative distribution function, and has the potential to relieve the often-debated exercise of determining an appropriate probability distribution function based on limited data, particularly in terms of tail behavior. Two numerical examples are provided for illustration.

Keywords: Exceedance Probability; Probability Bound; Distributionally Robust Optimization; Log-concave Distribution; Imprecise Probability

\footnotetext{
${ }^{1}$ Permanent postal address: Vestre Raa 50F, 5239 Raadal, Norway
} 


\section{Introduction}

The exceedance probability is the probability of an uncertain parameter exceeding a certain threshold. The complement of exceedance probability is often called the non-exceedance probability. The common approach for calculating the exceedance or non-exceedance probabilities is based on a description of uncertainty by a probability density function. Methods such as the method of moments or maximum likelihood are often used to estimate the parameters of an assumed or justified probability model based on a set of data points. With small exceedance probabilities in question, the choice of probability distribution is often a source of argument, especially if the amount of underlying data is not abundant.

The present paper builds on the alternative idea of working with a probability distribution class. A distribution class is the set of all probability distributions that satisfy a certain number of prior pieces of information as constraints. Such prior information can for instance be the distributional moment information, support of the distribution, or properties such as symmetry or unimodality for the probability distribution. The problem then becomes a matter of calculating the upper bound of exceedance probability, given any probability distribution belonging to this set. In this paper, this is called a "distributionally robust" approach, inspired by the terminology in the optimization community for problems involving uncertainty descriptions of a similar spirit-i.e., distributionally robust optimization. For example, see [1]. In civil and mechanical engineering domains, this perspective is often known and categorized under the theory of "imprecise probabilities"; see, e.g., [2] and [3].

The notion of evaluating the bounds of tail probabilities by relying on the distribution moments goes back to the $19^{\text {th }}$ century. For instance, the standard (one-sided) Chebyshev inequality provides an upper bound of the exceedance probability for a distribution class defined based on only the first two distribution moments (see Appendix A). Based on the same concept, several Chebyshev-like inequalities were developed in the late $19^{\text {th }}$ and early $20^{\text {th }}$ centuries, focusing on providing probability estimates for deviations from the mean, in terms of the moments, potentially also by considering additional information such as the unimodality of the distribution; a review of a number of such probability inequalities is summarized in [4]. Generalizations of the Chebyshev-like inequalities (e.g., to higher dimensions) were subjects of considerable research in the 1950s and 1960s. Some important examples belonging to this period are [5], [6], and [7]. These studies were based on the developments in mathematical optimization, specifically linear programming and duality theory, but were limited by the extents of optimization theory in those decades. The advances that followed in more recent years in mathematical optimization - namely, the introduction of interior-point methods and particularly semidefinite programs (SDPs) — resulted in their further developments; see, e.g., [8], [9], [10], [11], [12], and [13].

In this paper, a univariate class of distributions is introduced that builds on the first two distribution moments, in addition to a so-called log-concavity constraint for the cumulative distribution function (CDF) as prior information; see the next section for the definition of log-concavity. A one-sided Chebyshev-like inequality is then derived to calculate the upper bound of the exceedance probability given the constraints imposed by these priors. The recent methodology developed in [14] is used to solve the underlying optimization problem for this distribution class and derive the intended Chebychevlike inequality. The use of this class is motivated by two benefits:

1. One does not need to assume or establish any specific probability distribution. Instead, here, one only postulates that the probability distribution has a specific property, being that it is logarithmically concave in CDF. This implies a relevant constraint on the shape and tail behavior of potential candidate distributions. As will be seen in the paper, the assumption on log-concavity of the CDF has the power of incorporating a large number of commonly used parametric probability distributions and can be a natural choice.

2. In practice, one only needs to estimate the mean and the variance of a dataset. This is a straightforward and minimal exercise, which might allow for less controversy when dealing with datasets that do not contain a significant number of data points. Additionally, because the moment information is combined with a realistic assumption on the shape of the distribution (i.e., log-concavity of the $\mathrm{CDF}$ ), the proposed probability bound is considerably tighter than the bound obtained from the application of a standard one-sided Chebyshev inequality.

The paper is organized into five sections and two appendices. Section 2 gives some mathematical definitions before the main result is presented in Section 3. Section 4 presents two applications of the main result, while Section 5 concludes the work. Some technical results, including the proof of the main theorem, are presented in the appendices. 


\section{Preliminaries}

First, some preliminary definitions are given. The mathematically proficient reader can skip this section.

A function $f: \mathbb{R}^{n} \rightarrow \mathbb{R}$ is convex if

$f(\lambda \mathbf{x}+(1-\lambda) \mathbf{y}) \leq \lambda f(\mathbf{x})+(1-\lambda) f(\mathbf{y}) \quad \forall \mathbf{x}, \mathbf{y} \in \mathbb{R}^{n}$ and $\forall \lambda \in[0,1]$

and a function $f: \mathbb{R}^{n} \rightarrow \mathbb{R}$ is concave if $-f$ is convex. Graphically, a function is convex whose epigraph (the area above the curve/surface) has a convex geometry (or set of points), and a function is concave whose hypograph (the area below the curve/surface) has a convex geometry; see Fig. 1. The epigraph and hypograph of $f: \mathbb{R}^{n} \rightarrow \mathbb{R}$ are defined as

epi $f=\left\{(\mathbf{x}, t) \in \mathbb{R}^{n+1}: f(\mathbf{x}) \leq \mathrm{t}\right\}$
hyp $f=\left\{(\mathbf{x}, t) \in \mathbb{R}^{n+1}: f(\mathbf{x}) \geq \mathrm{t}\right\}$.
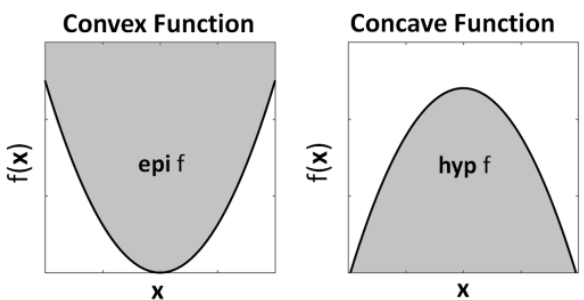

Fig. 1. Convex vs concave function $f: \mathbb{R} \rightarrow \mathbb{R}$.

Now, the concept of log-concavity is presented. A non-negative function $f: \mathbb{R}^{n} \rightarrow \mathbb{R}_{+}$is logarithmically concave or log-concave if

$f(\lambda \mathbf{x}+(1-\lambda) \mathbf{y}) \geq f(\mathbf{x})^{\lambda} \cdot f(\mathbf{y})^{1-\lambda} \quad \forall \lambda \in[0,1]$ and $\forall \mathbf{x}, \mathbf{y} \in \mathbb{R}^{n}$.

This is equivalent to saying that $f: \mathbb{R}^{n} \rightarrow \mathbb{R}_{+}$is $\log$-concave if $\log f$ is a concave function-i.e., if

$\log f(\lambda \mathbf{x}+(1-\lambda) \mathbf{y}) \geq \lambda \log (f(\mathbf{x}))+(1-\lambda) \log (f(\mathbf{y})) \quad \forall \lambda \in[0,1]$ and $\forall \mathbf{x}, \mathbf{y} \in \mathbb{R}^{n}$

with convention $\log 0=-\infty$.

A probability density function (PDF) or a cumulative distribution function (CDF) can be log-concave accordingly. It is known that any distribution with a log-concave PDF also has a log-concave CDF; see, e.g., [15]. However, the converse is not true and there are important probability distributions with log-concave CDF but not PDF. Table 1 lists a wide range of probability distributions and emphasizes whether their PDF and/or CDF are log-concave. The focus of this paper is on the log-concave CDF class, as it exclusively contains the log-concave PDF class but is relatively larger and contains the few distributions that slip from the PDF class-e.g., the lognormal distribution. In general, log-concavity is attributed with a wealth of appealing properties which all motivate their application; see, e.g., [15], [16], and [17]. However, this family is much more varied than for instance the Gaussian distribution, as it can admit to skewness, asymmetry, and a much more flexible description of uncertainty.

A number of distributions are selected and their PDF, logarithm of PDF, CDF, and logarithm of CDF are plotted in Fig. 2. The distribution functions plotted here are results of fitting to a set of data points based on the method of maximum likelihood. The dataset has a mean estimate of 3.7 and a standard deviation estimate of 0.923 . As seen from the logarithm plots, hypographs of the logarithm of all PDFs (except the last case: lognormal distribution) are convex regions, and hence the underlying PDFs are log-concave. Alternatively, the hypographs of the logarithm of the CDFs are all convex regions with no exception, which indicates that all the probability distributions are log-concave in CDF. 

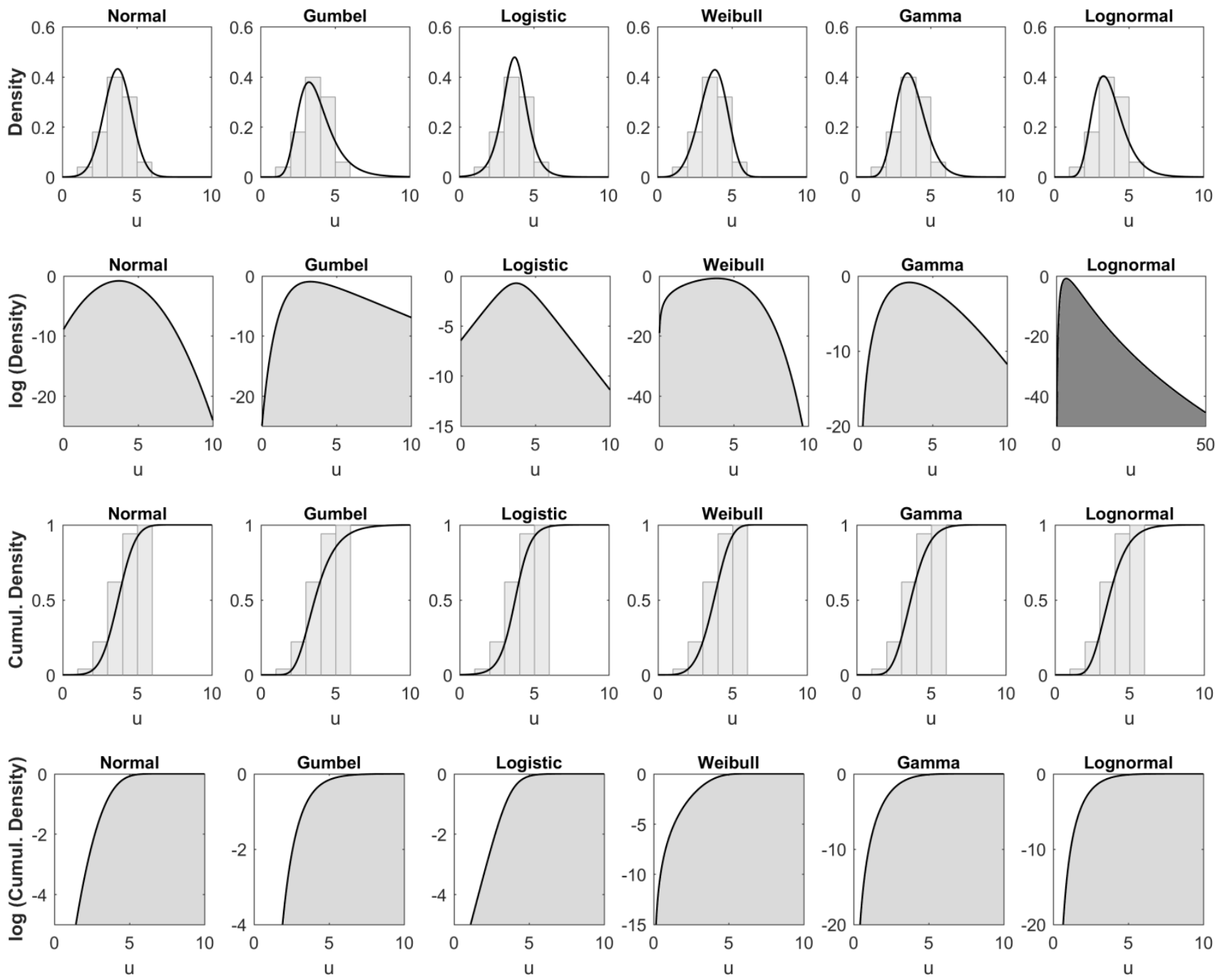

Fig. 2. Log-concavity of several univariate parametric distributions in terms of their PDF or CDF. Convex regions (and hence log-concave underlying PDF/CDF) are shaded with light gray where non-convex (and hence non-log-concave underlying $\mathrm{PDF} / \mathrm{CDF}$ ) are shaded with dark gray. 
Table 1. Log-concavity of a number of commonly used probability distributions.

\begin{tabular}{|c|c|c|c|c|c|}
\hline Class & & Distribution & Parameter Restriction & PDF & CDF \\
\hline \multirow{19}{*}{ 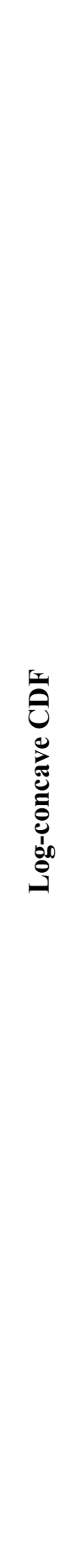 } & \multirow{14}{*}{ 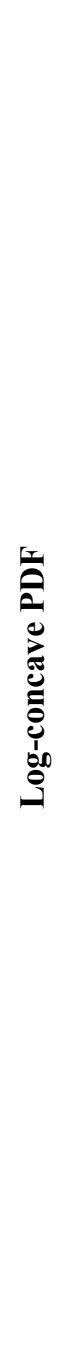 } & Normal & - & log-concave & log-concave \\
\hline & & Exponential & - & log-concave & log-concave \\
\hline & & Gumbel & - & log-concave & log-concave \\
\hline & & Laplace & - & log-concave & log-concave \\
\hline & & Logistic & - & log-concave & log-concave \\
\hline & & Rayleigh & - & log-concave & log-concave \\
\hline & & Maxwell & - & log-concave & log-concave \\
\hline & & Uniform & - & log-concave & log-concave \\
\hline & & Weibull ${ }^{1)}$ & shape parameter $\geq 1$ & log-concave & log-concave \\
\hline & & Gamma $^{2)}$ & shape paramter $\geq 1$ & log-concave & log-concave \\
\hline & & Beta & both parameters $\geq 1$ & log-concave & log-concave \\
\hline & & Power Function & parameter $\geq 1$ & log-concave & log-concave \\
\hline & & Chi-square & parameter $\geq 2$ & log-concave & log-concave \\
\hline & & Chi & parameter $\geq 1$ & log-concave & log-concave \\
\hline & \multirow{5}{*}{ 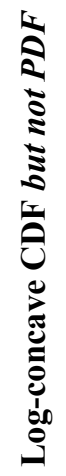 } & Lognormal & - & neither & log-concave \\
\hline & & Pareto & - & log-convex & log-concave \\
\hline & & Power Function & $0<$ parameter $<1$ & log-convex & log-concave \\
\hline & & Weibull $^{1)}$ & $0<$ parameter $<1$ & log-convex & log-concave \\
\hline & & Gamma $^{2)}$ & $0<$ parameter $<1$ & log-convex & log-concave \\
\hline \multirow[t]{2}{*}{ None } & & Student's t & - & neither & neither \\
\hline & & Cauchy & - & neither & neither \\
\hline
\end{tabular}

1) This is the three-parameter Weibull distribution with a location, a scale, and a shape parameter. As long as the shape parameter is restricted to values equal to or above one, both the PDF and CDF are log-concave. If the shape parameter lies between zero and one, the CDF is logconcave, but not the PDF. 
2) This is the three-parameter Gamma distribution with a location, a scale, and a shape parameter. As long as the shape parameter is restricted to values equal to or above one, both the PDF and CDF are log-concave. If the shape parameter lies between zero and one, the CDF is logconcave, but not the PDF.

\section{Main Result and Discussion}

\subsection{Formal definition}

The log-concave CDF class, which is the focus of this article, is formally defined here. Dealing with an uncertain quantity represented by a random variable $X \in \mathbb{R}$, such a class denoted by $\mathcal{P}_{\iota, \text { cdf }}\left(E X, \sigma(X)^{2}\right)$ is defined as the set of all probability distributions that:

1. Satisfy the first-moment information imposed by the mean/expected value $E X$;

2. Satisfy the second-moment information imposed by the variance value $\sigma(X)^{2}$; and,

3. Whose cumulative distribution functions are log-concave.

\subsection{Main Theorem}

The least upper bound or supremum probability of a random variable $X \in \mathbb{R}$ belonging to the log-concave CDF class $\mathcal{P}_{\iota, \text { cdf }}\left(E X, \sigma(X)^{2}\right)$ exceeding a certain threshold $\tilde{x}>E X$ is denoted by $\mathrm{E}_{\iota, \mathrm{cdf}}(\tilde{x})=p$ and can be computed by solving the equation

$$
\frac{\left(1+2 p \log p-p^{2}\right)^{0.5}}{p-\log p-1}=\frac{\sigma(X)}{\tilde{x}-E X} .
$$

The proof is based on the general methodology developed in [14] and is moved to Appendix A for better readability of this article.

\subsection{Discussion}

The function $\mathrm{E}_{\iota, \mathrm{cdf}}$ introduced in Section 3.2 is called the exceedance probability function in this paper. The inverse of this function is denoted $\mathrm{E}^{-1}{ }_{\iota, \mathrm{cdf}}:[0,1] \rightarrow \mathbb{R}$ which can be used to calculate the value of an uncertain quantity that corresponds to a maximum target exceedance level. It is common to represent the threshold $\tilde{x}$ as the mean value plus a multiple of the standard deviation-i.e., $\tilde{x}=E X+\gamma \sigma(X)$. In this case, the right-hand side of equation (5) will reduce to $\gamma^{-1}$. In other words, one can choose to work with a zero-mean standardized random variable $Z=(X-E X) / \sigma(X)$ represented by the class $\mathcal{P}_{\iota, \mathrm{cdf}}(0,1)$. This is a practical approach because the exceedance probability can in this case be represented as the function of a single parameter $\gamma$, which is the number of standard deviations away from the mean. This function is called the standard exceedance probability function and is denoted $\Lambda_{\iota, \mathrm{cdf}}(\gamma)$. The inverse of this function is denoted $\Lambda^{-1} \iota, \mathrm{cdf}:[0,1] \rightarrow \mathbb{R}$.

If the log-concave PDF class was of interest - let us denote it $\mathcal{P}_{\iota, \mathrm{pdf}}\left(E X, \sigma(X)^{2}\right)$ - then a relatively tighter bound for exceedance probability could be established. However, as discussed in Section 2, the log-concave PDF class is a smaller class, which for instance does not contain a handful of parametric distributions that the log-concave CDF class does. The bound corresponding to the log-concave PDF class is derived in [14] using the same methodology as used in this article for derivation of equation (5). Alternatively, if a class of distributions was defined based on the first two distribution moments and the condition on unimodality (rather than log-concavity) of the PDF, it would often result in a much looser bound. This is called the unimodal class and is denoted by $\mathcal{P}_{\star}\left(E X, \sigma(X)^{2}\right)$. For the explicit definition of the unimodal class and some discussions, see Appendix B. In this case, the result in [13] can be used to evaluate the exceedance probability bound. Defining a so-called Chebyshev class $\mathcal{P}_{\infty}\left(E X, \sigma(X)^{2}\right)$ only based on the first two moments and applying the one-sided version of the Chebyshev inequality [18] results in a yet looser bound. It is possible to define standard probability functions for all these classes denoted by $\Lambda_{\iota, \text { pdf }}, \Lambda_{\star}$, and $\Lambda_{\infty}$; see [19] for further discussions.

Obviously, if the underlying probability distribution were known, the exceedance probability function would become one minus the CDF of the particular distribution in question. For a Gaussian description of uncertainty, it is possible to define the standard exceedance probability function $\Lambda_{\text {Gaussian }}$ and evaluate it as $\Lambda_{\text {Gaussian }}=1-\Phi(\gamma)=\Phi(-\gamma)=p$ where 
$\Phi$ is the standard cumulative distribution function of the Gaussian distribution. Taking the inverse, $\gamma$ can be calculated as $\gamma=-\Phi^{-1}(p)=-\Lambda^{-1}$ Gaussian, which coincides with the reliability index in the structural reliability literature; see, e.g., [20]. In fact, by the proposed methodology in this article, a safe estimate of the reliability index (or probability of failure/exceedance) can be calculated for a versatile range of probability distributions by targeting the log-concave $\mathrm{CDF}$ class, when a univariate uncertainty (e.g., in action effect) and a given threshold is in question.

It might be interesting to mention that for the distributionally robust philosophy-e.g., involving the log-concave CDF class, log-concave PDF class, unimodal class, or Chebyshev class-function $\Lambda($.$) can be thought of as a cumulative$ belief function, considering a Dempster-Shafer structure. A further discussion of this interpretation is considered to be out of scope of this paper.

Standard exceedance function $\Lambda($.$) is plotted in Fig. 3$ for the log-concave CDF class. Additionally, it is contrasted to the corresponding case for normal distribution, as well as the log-concave PDF, unimodal and Chebyshev classes for comparison. In this figure, $1 / \gamma$ is used on the horizontal axis to highlight the tail behavior of the different classes/distributions.

As can be inferred from Fig. 3 and briefly discussed earlier, tightness of the estimated probability bound is directly linked to the strength of prior assumptions regarding the underlying uncertainty. The bound provided by the one-sided Chebyshev inequality is only based on the first two moments and no additional information, which is attained by a pessimistic discrete distribution with masses concentrated in the upper and lower tails. Such a result is often physically unlikely, particularly when considering small exceedance probabilities; see, e.g., the numerical example in Section 4.1. Incorporating the additional unimodality condition improves the probability bound and is a reasonable assumption in most practical situations. For instance, it is reasonable (and common practice) to assume that stronger winds, waves, earthquakes, and floods are attributed with smaller probabilities of occurrence-i.e., the probability distribution that describes them is unimodal. This behavior is not recognized by the application of the Chebyshev class. However, the strength of the unimodality condition might not yet be sufficient to yield reasonable bounds for these physical quantities, particularly when small exceedance probabilities are in question. This is the background to propose the much stronger logconcavity condition (e.g. on the $\mathrm{CDF}$ ), because the commonly used probability distributions mostly share this property. This assumption would tighten the probability bound much further, and would make it sufficiently close to the bounds obtained by the application of the frequently used probability distributions. This is better understood by following the numerical examples in Section 4.

With respect to the effect of strength of prior assumption on tightness of the probability bound, it might also be interesting to mention the former results in [21] where uncertainty was only described by the first-moment condition and the log-concavity of the CDF, but no second-moment information. In this simpler case, the exceedance probability can be calculated according to equation (6). It can be shown that this is generally a much looser bound than the one provided in equation (5) and can become even looser than the Chebyshev bound, depending on the value of the moment(s) and the threshold in question. This is the penalty for eliminating the second-moment information, or in other words working with a much weaker prior piece of information.

$\mathrm{E}_{\iota, \operatorname{cdf}}(\tilde{x})=1-e^{-1}\left(\frac{\tilde{x}}{\tilde{x}-E X}\right)^{\frac{\tilde{x}}{E X}-1}$ 


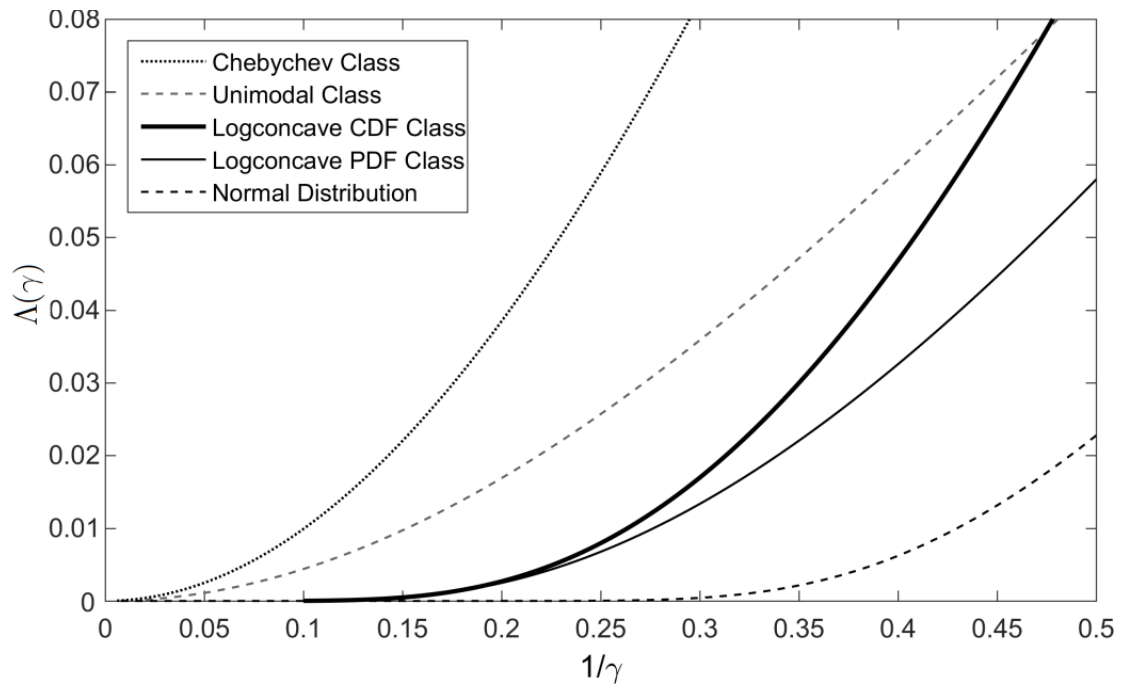

Fig. 3. Function $\Lambda(\gamma)$ for different probability classes, compared to the Gaussian/normal distribution.

\section{Numerical Examples}

\subsection{Estimation of Environmental Parameters}

The problem of finding the value of physical environmental quantities that comply with a target annual exceedance probability level occurs frequently in offshore engineering applications. Such environmental parameters can, for instance, be the characteristics of the wind (e.g., speed), wave (e.g., significant height and spectral period), current (e.g., speed) and sea ice (e.g., thickness and strength). This example treats the sea ice as the environmental parameter of interest. Sixty-three years of annual maximum sea-ice thickness data are considered here based on measurements related to Norströmsgrund lighthouse in the Baltic Sea. The data are taken from [22] and are plotted in the form of a bar-plot in Fig. 4, where the first bar corresponds to the year from winter 1952 to winter 1953. In the plot, the mean level as well as the mean plus and minus one standard deviation levels are highlighted with dashed lines. The estimated mean of the sample is $\mu=0.66 \mathrm{~m}$ and its standard deviation is $\sigma=0.175 \mathrm{~m}$.

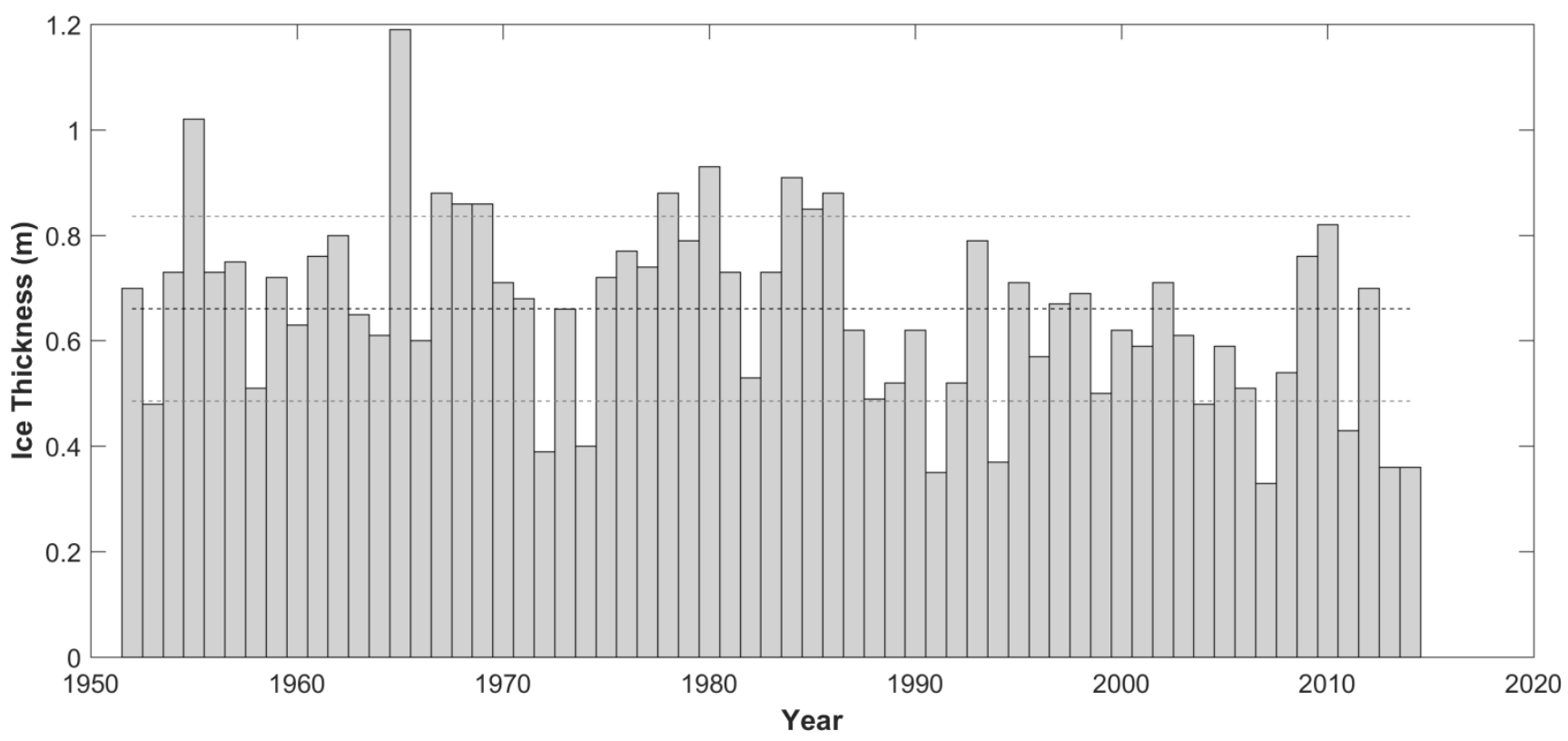

Fig. 4. Annual maximum ice thickness data from 1952 to 2015.

Several parametric distributions were fitted to the annual maximum data being normal, Gumbel, Weibull, gamma, logistic and lognormal; see Fig. 5, Fig. 6, and Fig. 7. In this regard, method of maximum likelihood was used for estimation of distribution parameters. Accordingly, the ice thicknesses corresponding to annual exceedance probability 
levels of $10^{-1}, 10^{-2}$, and $10^{-3}$, often referred to as 10-year, 100-year, and 1000-year values, are inferred and listed in Table 2 .

The log-concave CDF class being the focus of this article is also applied. As seen from the table of results, the 10-year, 100 -year and 1000-year values based on this class nicely bound the corresponding values from all postulated probability distributions, which are all log-concave in CDF and hence belong to the class. This can also be inferred by studying Fig. 6 and Fig. 7. For instance, the 1000-year value, which is often an important design value, is $1.70 \mathrm{~m}$ based on the logconcave CDF class where among the tested probability distributions Gumbel provides the worst value of $1.69 \mathrm{~m}$.

The log-concave PDF class is also considered as a subclass of the log-concave CDF class. The results provided by the log-concave PDF class also essentially bound the results of the tested probability distributions. However, small violations occur in this case for the 10-year and 100-year values compared to the value yielded by application of the Gumbel distribution. This is in spite of the fact that the Gumbel distribution is log-concave in PDF. Even though small, this is attributed to the uncertainty in the moment estimates when using this distribution class. One should also note that the estimated distribution parameters (e.g., of the Gumbel distribution) are also subject to uncertainty and are not definite. This is resolved by applying the $95 \%$ confidence upper bound value of the mean estimate, resulting in upper bound values that bound the Gumbel 10-year and 100-year estimates. Values based on such a 95\% estimate are listed in parentheses in the table of results. The $95 \%$ confidence interval is calculated using the sample estimates of the mean and standard deviation, by applying the t-distribution for the sampling distribution of the sample mean.

More relaxed unimodal and Chebyshev classes are also examined, resulting in generally larger estimates. These estimates rapidly diverge from the results obtained from the log-concave CDF class or the known probability distributions as the target annual exceedance probability level decreases. For instance, the 1000-year thickness value based on the application of Chebyshev class is $6.2 \mathrm{~m}$, which appears to be exaggeratedly large, and physically unlikely.

The fact that the Gumbel distribution stands out among the examined parametric distributions is rather arbitrary. By perturbing the data points to a slight degree, other distributions such as lognormal became dominant. The results of this artificial sensitivity study are not reported here.

Table 2. 10-year, 100-year, and 1000-year ice thickness values.

\begin{tabular}{|c|c|c|c|}
\hline \multirow{2}{*}{ Distribution/Class } & \multicolumn{3}{|c|}{ Ice Thickness (m) } \\
\hline & 10-Year Value & 100-Year Value & 1000-Year Value \\
\hline Normal & 0.88 & 1.07 & 1.20 \\
\hline Gumbel (Extreme Value Type I) & 0.94 & 1.32 & 1.69 \\
\hline Weibull (Extreme Value Type III) & 0.89 & 1.05 & 1.16 \\
\hline Gamma & 0.90 & 1.15 & 1.35 \\
\hline Logistic & 0.88 & 1.12 & 1.34 \\
\hline Lognormal & 0.91 & 1.22 & 1.52 \\
\hline Log-concave PDF Class & $0.93(0.98)$ & $1.29(1.34)$ & $1.70(1.74)$ \\
\hline Log-concave CDF Class & $1.00(1.04)$ & $\mathbf{1 . 3 3}(1.37)$ & $1.70(1.75)$ \\
\hline Unimodal Class & $0.98(1.02)$ & $1.82(1.86)$ & $4.36(4.40)$ \\
\hline Chebyshev Class & $1.19(1.23)$ & $2.40(2.45)$ & $6.20(6.25)$ \\
\hline
\end{tabular}

Note: Values in parentheses are based on $95 \%$ confidence upper bound value of the mean and are therefore slightly larger than the un-bracketed values.

In offshore and in particular Arctic offshore engineering applications, sixty-three years of data are considered a long time series that are not often available for most geographical locations of interest. Here, the applicability of the method is 
illustrated when a limited number of years of data are available and the development of the obtained estimates is shown as the amount of information increases. In this respect, the entire dataset is divided into ten batches, each consisting of six years of data except the last one that contains nine years of data. The first and second moments of the data are then estimated for the first batch and the 10-, 100-, and 1000-year estimates of ice thickness are calculated for the log-concave CDF class. The estimates are either obtained based on the point estimate of the sample mean or alternatively by applying the $95 \%$ confidence upper bound estimate of the sample mean. This procedure is repeated in a cumulative fashion by including the additional batches of data until the entire dataset is covered. The results are listed in Table 3, Table 4, and Table 5. Interestingly, the obtained estimates of 10-, 100-, and 1000-year ice thickness using this distribution class to a great extent bound the values derived based on parametric distributions mentioned earlier, right from the first six-year batch up to the final year. Similar to before, the provided bounds are reasonably tight, which is the background for advocating the log-concave CDF class.

It is worth mentioning that the probability distribution of ice thickness might be non-stationary-i.e., it might change-in time. For instance, a lognormal distribution may be a good fit to the first six years of data, whereas when the amount of data increases - e.g., to twelve years - another type of distribution such as Gumbel may be a better fit. In the distributionally robust methodology, stationarity in time in fact means that the probability distribution must remain in the specified distribution class (here, the specified log-concave CDF class) as time goes by, and the provided probability bound is the upper bound of exceedance probability given any precise probability distribution from the specified class. In other words, it not required that the precise probability distribution type remain unchanged in time, but that the probability distribution class remain unchanged in time. However, it is implicitly required that the distribution moments be stationary in time. The above exercise illustrates the relative stability of the probability bound as time goes by in this example. This may be interpreted as an indication of a reasonably well performance of the distributionally robust philosophy, in view of a potential non-stationarity of distribution type in time.

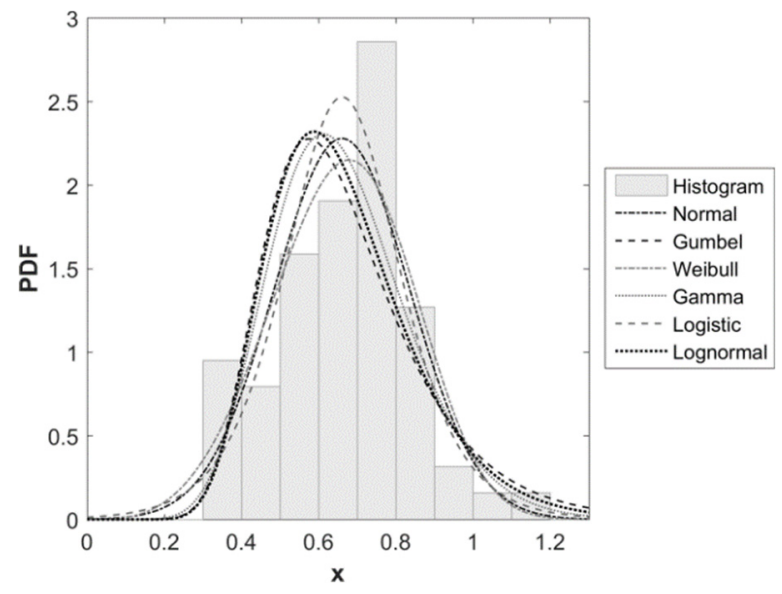

Fig. 5. PDFs of the fitted distributions to the sixty-three-year annual maximum data.

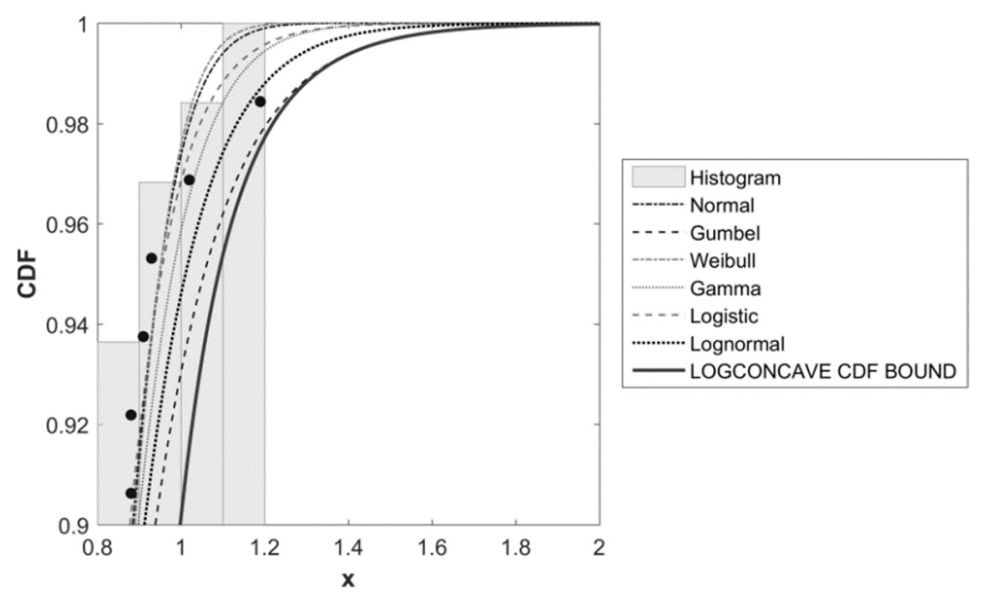

Fig. 6. CDFs of the fitted distributions contrasted against the log-concave CDF bound. 


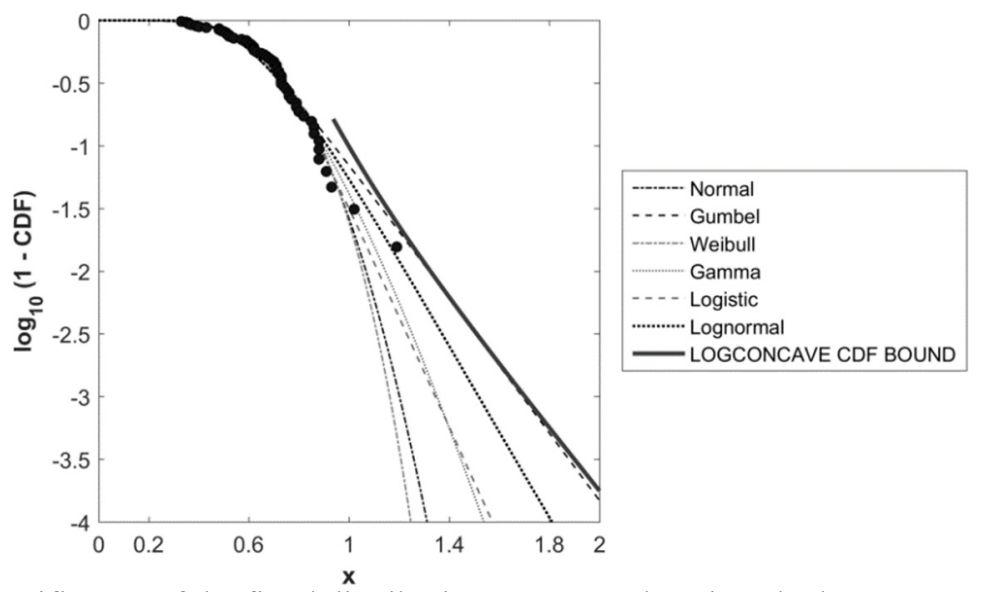

Fig. 7. Tail significance of the fitted distributions contrasted against the log-concave CDF bound.

Table 3. Development of 10-year ice thickness values as the amount of data increases.

10-Year Ice Thickness Based on the Log-concave CDF Class

\begin{tabular}{cccccccccccc}
\hline Estimation Method & $\begin{array}{c}\text { Year 1 } \\
\text { to 6 }\end{array}$ & $\begin{array}{c}\text { Year 1 } \\
\text { to 12 }\end{array}$ & $\begin{array}{c}\text { Year 1 } \\
\text { to 18 }\end{array}$ & $\begin{array}{c}\text { Year 1 } \\
\text { to 24 }\end{array}$ & $\begin{array}{c}\text { Year 1 } \\
\text { to 30 }\end{array}$ & $\begin{array}{c}\text { Year 1 } \\
\text { to 36 }\end{array}$ & $\begin{array}{c}\text { Year 1 } \\
\text { to 42 }\end{array}$ & $\begin{array}{c}\text { Year 1 } \\
\text { to 48 }\end{array}$ & $\begin{array}{c}\text { Year 1 } \\
\text { to 54 }\end{array}$ & $\begin{array}{c}\text { Year 1 } \\
\text { to 63 }\end{array}$ \\
\hline $\begin{array}{c}\text { Point Estimate of the } \\
\text { Sample Mean }\end{array}$ & 1.07 & 0.97 & 1.08 & 1.06 & 1.05 & 1.05 & 1.04 & 1.02 & 1.00 & $\mathbf{1 . 0 0}$ \\
$\begin{array}{c}95 \% \text { Confidence Upper } \\
\text { Bound Estimate of the } \\
\quad \text { Sample Mean }\end{array}$ & 1.25 & 1.06 & 1.17 & 1.13 & 1.12 & 1.10 & 1.09 & 1.07 & 1.05 & $\mathbf{1 . 0 4}$ \\
\hline
\end{tabular}

Table 4. Development of 100-year ice thickness values as the amount of data increases.

100-Year Ice Thickness Based on the Log-concave CDF Class

\begin{tabular}{|c|c|c|c|c|c|c|c|c|c|c|}
\hline Estimation Method & $\begin{array}{l}\text { Year } 1 \\
\text { to } 6\end{array}$ & $\begin{array}{l}\text { Year } 1 \\
\text { to } 12\end{array}$ & $\begin{array}{l}\text { Year } 1 \\
\text { to } 18\end{array}$ & $\begin{array}{l}\text { Year } 1 \\
\text { to } 24\end{array}$ & $\begin{array}{l}\text { Year } 1 \\
\text { to } 30\end{array}$ & $\begin{array}{l}\text { Year } 1 \\
\text { to } 36\end{array}$ & $\begin{array}{l}\text { Year } 1 \\
\text { to } 42\end{array}$ & $\begin{array}{l}\text { Year } 1 \\
\text { to } 48\end{array}$ & $\begin{array}{l}\text { Year } 1 \\
\text { to } 54\end{array}$ & $\begin{array}{l}\text { Year } 1 \\
\text { to } 63\end{array}$ \\
\hline $\begin{array}{l}\text { Point Estimate of the } \\
\text { Sample Mean }\end{array}$ & 1.39 & 1.23 & 1.41 & 1.39 & 1.37 & 1.35 & 1.36 & 1.34 & 1.31 & 1.33 \\
\hline $\begin{array}{l}95 \% \text { Confidence Upper } \\
\text { Bound Estimate of the } \\
\text { Sample Mean }\end{array}$ & 1.57 & 1.32 & 1.49 & 1.47 & 1.43 & 1.41 & 1.42 & 1.39 & 1.36 & 1.37 \\
\hline
\end{tabular}

Table 5. Development of 1000-year ice thickness values as the amount of data increases.

1000-Year Ice Thickness Based on the Log-concave CDF Class

\begin{tabular}{|c|c|c|c|c|c|c|c|c|c|c|}
\hline Estimation Method & $\begin{array}{l}\text { Year } 1 \\
\text { to } 6\end{array}$ & $\begin{array}{l}\text { Year } 1 \\
\text { to } 12\end{array}$ & $\begin{array}{l}\text { Year } 1 \\
\text { to } 18\end{array}$ & $\begin{array}{l}\text { Year } 1 \\
\text { to } 24\end{array}$ & $\begin{array}{l}\text { Year } 1 \\
\text { to } 30\end{array}$ & $\begin{array}{l}\text { Year } 1 \\
\text { to } 36\end{array}$ & $\begin{array}{l}\text { Year } 1 \\
\text { to } 42\end{array}$ & $\begin{array}{l}\text { Year } 1 \\
\text { to } 48\end{array}$ & $\begin{array}{l}\text { Year } 1 \\
\text { to } 54\end{array}$ & $\begin{array}{l}\text { Year } 1 \\
\text { to } 63\end{array}$ \\
\hline $\begin{array}{c}\text { Point Estimate of the } \\
\text { Sample Mean }\end{array}$ & 1.76 & 1.53 & 1.78 & 1.78 & 1.73 & 1.71 & 1.73 & 1.71 & 1.67 & 1.70 \\
\hline $\begin{array}{l}95 \% \text { Confidence Uppe } \\
\text { Bound Estimate of the } \\
\text { Sample Mean }\end{array}$ & 1.94 & 1.62 & 1.87 & 1.85 & 1.79 & 1.76 & 1.79 & 1.76 & 1.71 & 1.75 \\
\hline
\end{tabular}




\subsection{Structural Reliability Application}

A timber beam of length $5 \mathrm{~m}$ is in question. If the action effect in the beam $S$ is treated as an uncertain parameter with mean of $3.75 \mathrm{MN}$ and variance of $0.55 \mathrm{MN}^{2}$, what should the beam resistance be (in a deterministic sense) to have $10^{-2}$ or $10^{-3}$ in maximum probability of failure $\mathrm{P}_{\mathrm{f}}$ ?

The safety margin in this problem is defined as $g(r, S)=r-S$ and the aim of analysis is to find the value of $r$ such that $\operatorname{Prob}(g(r, S)<0) \leq 10^{-2}$ or $10^{-3}$.

The problem is solved for normal, gamma and lognormal distributions, parameters of which can be deduced based on the given mean and variance. Additionally, the log-concave CDF class, unimodal class and the Chebyshev class are considered. As seen from Table 6, the required resistance by applying the log-concave CDF class provides reasonable bounds to those provided by the studied parametric probability distributions (and definitely many more that are not considered). However, the application of the unimodal and Chebyshev classes seems to push the limits in particular when the target probability of failure decreases.

In structural reliability problems where more than one uncertain parameter is involved, it may be of interest to describee.g., one of the uncertain parameters for which less amount of data is available - with a log-concave CDF class, and the rest by known probability distribution functions. In this practical scenario, the algorithm provided in [19] can be used, which combines the power of a traditional Monte Carlo simulation with the methodologies such as those presented in this article. This is left outside the scope of this article for brevity.

Table 6. Required resistance based on different assumptions for description of uncertainty.

\begin{tabular}{|c|c|c|c|c|}
\hline \multirow[b]{2}{*}{ Distribution/Class } & \multicolumn{2}{|c|}{$\mathrm{P}_{\mathrm{f}}: 10^{-2}$} & \multicolumn{2}{|c|}{$\mathrm{P}_{\mathrm{f}}: 10^{-3}$} \\
\hline & $\gamma$ & $\begin{array}{c}\text { Resistance } \\
(\mathrm{MN})\end{array}$ & $\gamma$ & $\begin{array}{c}\text { Resistance } \\
(\mathrm{MN})\end{array}$ \\
\hline Normal & 2.33 & 5.48 & 3.09 & 6.04 \\
\hline Gamma & - & 5.59 & - & 6.46 \\
\hline Lognormal & - & 5.80 & - & 6.74 \\
\hline Log-concave CDF Class & 3.80 & 6.56 & 5.95 & 8.16 \\
\hline Unimodal Class & 6.60 & 8.86 & 21.10 & 19.40 \\
\hline Chebyshev Class & 9.95 & 11.13 & 31.61 & 27.19 \\
\hline
\end{tabular}

\section{Conclusions}

A one-sided Chebyshev-like inequality is derived for a class of distributions that covers the major probability distributions commonly used in civil, mechanical, and marine engineering applications. This class builds on the first two distribution moments, as well as a so-called log-concavity constraint on the CDF of the underlying probability distribution. The derivation can be used to calculate the upper bound of the so-called exceedance probability-i.e., the probability that an uncertain quantity exceeds a given limit. The novel feature of the method is that it is robust with respect to the choice of probability distribution, which in many practical situations is a matter of hesitation when dealing with far tail evaluations. At the same time, due to the relevance of the log-concavity constraint, the provided probability bound is considerably tighter than the bound obtained from the application of a one-sided Chebyshev inequality or even a one-sided version of Gauss inequality. As illustrated by examples, the method is able to produce tight probability bounds even with a limited number of data points - e.g., short duration of ocean environmental measurements. The method can also be applied in structural reliability applications where the number of data points for establishing a reliable probability distribution function (e.g., for particular material properties or load characteristics) is not promising. 


\section{APPENDIX A: Proof of Main Theorem}

A proof of the major result of this paper, equation (5), is given in this appendix. In the body of the paper, a measuretheoretic representation of probability has been avoided to increase the readability for application-oriented readers. The background material for reading this appendix can be found, for example, in [23]. Otherwise, simply neglect the next paragraph and then read $\mathbb{P}[A]$ as $\operatorname{Prob}[A]$ or probability of $A$ in the proofs that follow.

A random variable (r.v.) is any measurable function $X:(\Omega, \mathcal{M}, \mathbb{P}) \rightarrow \mathbb{R}$, where $(\Omega, \mathcal{M}, \mathbb{P})$ is a probability space, with $\mathcal{M}$ being a field of subsets of set $\Omega$, and $\mathbb{P}$ being a probability measure on $(\Omega, \mathcal{M})$. Let $F_{X}(x)=\mathbb{P}[X \leq x]$ denote the CDF of an r.v. $X$. It is assumed that the probability space $\Omega$ is atomless, i.e., there exist r.v.s on $\Omega$ with all possible CDFs (see, e.g., [24]). Attention is restricted to r.v.s from $\mathscr{L}^{p}(\Omega), 1 \leq p<\infty$, which is a Banach space with the norm $\|X\|_{p}=\left(E[|X| p)^{1 / p}\right.$.

It is often important to estimate the probability that an r.v. is significantly smaller, or significantly larger than its expected value-i.e., $\mathbb{P}[X \leq E X-a]$ or $\mathbb{P}[X \geq E X+a]$ for some $a>0$. Following any of these forms would yield identical results, even though the practical focus of this article is on the latter probability-i.e., the so-called exceedance probability. For the derivations that follow, $\mathbb{P}[X \leq E X-a]$ is used as the basis.

In terms of standard deviation $\sigma(X)=\|X-E X\|_{2}$, the probability $\mathbb{P}[X \leq E X-a]$ can be estimated using the standard (one-sided) Chebyshev inequality

$\mathbb{P}[X \leq E X-a] \leq \frac{\sigma(X)^{2}}{a^{2}+\sigma(X)^{2}}$

The functional $\sigma$ is a way to measure non-constancy of r.v. $X$ and is a special case of so-called general deviation measures [25, 26]. In this relation, [14] developed a methodology allowing to derive the best possible Chebyshev-type inequalities in the form

$\mathbb{P}[X \leq E X-a] \leq f_{D}(\mathcal{D}(X), a)$,

where $\mathcal{D}(X)$ is a deviation measure (as will be immediately defined). Additionally, [14] showed that inequalities such as (A.7) and (A.8) can be improved if $X$ is known to belong to specific families of distributions. For instance, the developed methodology has been applied to derive Chebyshev-type inequalities for (a) r.v.s with symmetric distribution, and (b) r.v.s with log-concave probability distribution function (PDF). As discussed, the purpose of this article is to have a similar inequality for the r.v.s with log-concave cumulative distribution function (CDF). Now, deviation measures will be defined.

Definition 1 (deviation measures) A deviation measure $[25,26]$ is any functional satisfying $\mathcal{D}: \mathscr{L}^{p}(\Omega) \rightarrow[0, \infty]$ $(\mathcal{D} 1) \mathcal{D}(X)=0$ for constant $X$, but $\mathcal{D}(X)>0$ otherwise (non-negativity).

$(\mathcal{D} 2) \mathcal{D}(\lambda X)=\lambda \mathcal{D}(X)$ for all $X$ and all $\lambda>0$ (positive homogeneity).

$\left(\mathcal{D}_{3}\right) \mathcal{D}(X+Y) \leq \mathcal{D}(X)+\mathcal{D}(Y)$ for all $X$ and $Y$ (sub-additivity).

$\left(\mathcal{D}_{4}\right)$ set $\left\{X \in \mathscr{L}^{p}(\Omega) \mid \mathcal{D}(X) \leq \mathrm{c}\right\}$ is closed for all $c<\infty$ (lower-semicontinuity).

Axioms $\mathcal{D}_{1}-\mathcal{D}_{3}$ imply [26], that

$\mathcal{D}(X+C)=\mathcal{D}(X)$ for all constants $C$ (insensitivity to constant shift).

Examples of deviation measures include standard deviation $\left(\sigma(X)=\|X-E X\|_{2}\right)$, lower and upper semi-deviations $\left(\sigma_{-}(X)=\left\|[X-E X]_{-}\right\|_{2}\right.$ and $\sigma_{+}(X)=\left\|[X-E X]_{+}\right\|_{2}$, where $[X]_{-}=\max \{0,-X\}$ and $\left.[X]_{+}=\max \{0, X\}\right)$, mean absolute deviation ( $M A D(X)=E|X-E X|)$, conditional value-at-risk (CVaR) deviation defined by

$\operatorname{CVaR}_{\alpha}^{\Delta}(X) \equiv E X-\frac{1}{\alpha} \int_{0}^{\alpha} q_{X}(\beta) d \beta, \alpha \in(0,1)$,

where $q_{X}(r)=\inf \left\{z \mid F_{X}(z)>r\right\}$, and many others; see [26]. 
A deviation measure $\mathcal{D}$ is called law-invariant if $\mathcal{D}(X)=\mathcal{D}(Y)$ whenever $X$ and $Y$ have the same CDF.

All the examples listed above are law-invariant deviation measures.

Now let $V$ be the set of all r.v.s with log-concave CDF. Our aim is, given any law-invariant deviation measure $\mathcal{D}$ (and in particular standard deviation), derive the best possible Chebyshev inequality (A.8) valid for all $X \in V$. Proposition 7 in [14] implies that $f_{D}$ in (A.8) is given by

$f_{D}(d, a)=\sup \left\{p \mid u_{\mathcal{D}}(p, a) \leq d\right\}$

where

$u_{\mathcal{D}}(p, a)=\inf _{X} \mathcal{D}(\mathrm{X})$

s.t. $X \in C_{p}=\left\{X \in \mathcal{L}^{p}(\Omega) \mid X \in V, \mathbb{P}[X \leq E X-a] \geq p\right\}$.

It is said that r.v. $X$ dominates r.v. $Y$ with respect to concave ordering, and write $X \succeq_{c} Y$ if $E X=E Y$ and

$$
\int_{-\infty}^{x} F_{X}(t) d t \leq \int_{-\infty}^{x} F_{Y}(t) d t
$$

for all $x \in \mathbb{R}$. Theorem 3 in [27] implies that a sufficient condition for $X \succeq_{c} Y$ is $E X=E Y$ and the existence of $x^{*} \in \mathbb{R}$ such that

$F_{X}(x) \leq F_{Y}(x)$ for $x \leq x^{*}$, and $F_{Y}(x) \geq F_{X}(x)$ for $x \geq x^{*}$

A set $U_{c} \subseteq C_{p}$ is called reduced set in (A.12), if for any $Y \in C_{p}$, there exists $X \in U_{c}$ such that $X \succeq_{c} Y$. Because for any law-invariant deviation measure $\mathcal{D}, X \succeq_{c} Y$ implies $\mathcal{D}(X) \leq \mathcal{D}(Y)$ [14], (A.12) is equivalent to

$u_{\mathcal{D}}(p, a)=\inf _{X \in U_{c}} \mathcal{D}(\mathrm{X})$

for any reduced set $U_{c}$.

Proposition 1 For any $a>0, p \in(0,1)$, one-parameter family $U_{c}$ of r.v.s $X$ with CDF

$$
F_{X}(x)= \begin{cases}0 & x<x_{0} \\ \exp \left[\frac{p-\log p-1}{a}\left(x-x_{0}+\frac{a \log p}{p-\log p-1}\right)\right] & x_{0} \leq x<x_{0}-\frac{a \log p}{p-\log p-1} \\ 1 & x_{0}-\frac{a \log p}{p-\log p-1} \leq x\end{cases}
$$

$x_{0} \in \mathbb{R}$, is a reduced set in (A.12).

Proof. First note that $p-\log p-1>0$ for any $p \in(0,1)$, hence $F_{X}(x)$ in (A.16) is well-defined. With $k=\frac{p-\log p-1}{a}>0, x_{1}=x_{0}-\frac{\log p}{k}=x_{0}-\frac{a \log p}{p-\log p-1}$, one obtains $F_{X}(x)=\exp \left[k\left(x-x_{1}\right)\right]$, $x_{0} \leq x<x_{1}$, hence the probability density function $f_{X}(x)=k \exp \left[k\left(x-x_{1}\right)\right], x_{0} \leq x<x_{1}$, and 


$$
\begin{aligned}
E X & =x_{0} \mathbb{P}\left[X=x_{0}\right]+\int_{x_{0}}^{x_{1}} x f_{X}(x) d x=x_{0} F_{X}\left(x_{0}\right)+\int_{x_{0}}^{x_{1}} x k e^{k\left(x-x_{1}\right)} d x=x_{0} p+\left.e^{-k x_{1}} k \frac{e^{k x}}{k^{2}}(k x-1)\right|_{x_{0}} ^{x_{1}}= \\
& =x_{0} p+\frac{k x_{1}-1}{k}-e^{k\left(x_{0}-x_{1}\right)} \frac{k x_{0}-1}{k}=x_{0} p+\frac{k x_{0}-\log p-1}{k}-p \frac{k x_{0}-1}{k}= \\
& =x_{0} p+\frac{k x_{0}-p k x_{0}+(p-\log p-1)}{k}=x_{0} p+\frac{k x_{0}-p k x_{0}+k a}{k}=x_{0}+a .
\end{aligned}
$$

Hence, $\mathbb{P}[X \leq E X-a]=\mathbb{P}\left[X \leq x_{0}\right]=F_{X}\left(x_{0}\right)=p$.

Inequality (3) for $f=F_{X}$ reduces to $F_{X}(\lambda \mathrm{x}+(1-\lambda) y) \geq 0$ if $\min \{x, y\}<x_{0}$, and follows from concavity of function

$\log F_{X}(x)= \begin{cases}k\left(x-x_{1}\right) & x_{0} \leq x<x_{1} \\ 0 & x_{1} \leq x\end{cases}$

otherwise. Hence, $X \in V$, and therefore $X \in C_{p}$ for every $x_{0} \in \mathbb{R}$. This implies that $U_{c} \subseteq C_{p}$.

Next, for any r.v. $Y \in C_{p}$ let us prove that $X \succeq_{c} Y$ for r.v. $X \in U_{c}$ corresponding to $x_{0}=E Y-a$. Then, $F_{Y}\left(x_{0}\right)=\mathbb{P}\left[Y \leq x_{0}\right]=\mathbb{P}[Y \leq E Y-a] \geq p=F_{X}\left(x_{0}\right)$, hence $\log \left[F_{Y}\left(x_{0}\right)\right] \geq \log \left[F_{X}\left(x_{0}\right)\right]$. On the other hand, $\log \left[F_{Y}\left(x_{1}\right)\right] \leq \log \left[F_{X}\left(x_{1}\right)\right]=0$. Because functions $\log \left[F_{Y}(x)\right]$ and $\log \left[F_{X}(x)\right]$ are concave and linear on $\left[x_{0}, x_{1}\right]$, respectively, there exist $x^{*} \in \mathbb{R}$ such that $\log F_{X}(x) \leq \log F_{Y}(x)$ for $x<x^{*}$, and $\log F_{Y}(x) \geq \log F_{X}(x)$ for $x \geq x^{*}$.

By monotonicity of logarithm, this implies (A.14), hence $X \succeq_{c} Y$.

Let $X_{0}$ be an r.v. with CDF (A.16) with $x_{0}=0$. By (A.9), $\mathcal{D}(X)=\mathcal{D}\left(X_{0}\right)$ for any $X \in U_{c}$, and (A.15) reduces to $u_{\mathcal{D}}(p, a)=\mathcal{D}\left(X_{0}\right)$.

Given the above, the main result of this article can now be presented.

Problem For any $a>0$, all r.v.s $X$ with log-concave CDF satisfy the Chebyshev inequality

$$
\mathbb{P}[X \leq E X-a] \leq p
$$

where $p \in(0,1)$ is the solution to the equation

$$
\frac{\left(1+2 p \log p-p^{2}\right)^{0.5}}{p-\log p-1}=\frac{\sigma(X)}{a}
$$

Detail. In notation as in proof of Proposition $1, x_{0}=0, k=\frac{p-\log p-1}{a}>0, x_{1}=-\frac{\log p}{k}$, one obtains

$$
\begin{aligned}
E\left[X_{0}^{2}\right] & =x_{0}^{2} \mathbb{P}\left[X=x_{0}\right]+\int_{x_{0}}^{x_{1}} x^{2} f_{X}(x) d x=\int_{0}^{x_{1}} x^{2} k e^{k\left(x-x_{1}\right)} d x=\left.e^{-k x_{1}} k e^{k x}\left(\frac{x^{2}}{k}-\frac{2 x}{k^{2}}+\frac{2}{k^{3}}\right)\right|_{0} ^{x_{1}}= \\
& =k\left(\frac{x_{1}^{2}}{k}-\frac{2 x_{1}}{k^{2}}+\frac{2}{k^{3}}\right)-k e^{-k x_{1}} \frac{2}{k^{3}}=\frac{\log ^{2}(p)}{k^{2}}+\frac{2 \log p}{k^{2}}+\frac{2}{k^{2}}-p \frac{2}{k^{2}}= \\
& =a^{2} \frac{\log ^{2}(p)+2 \log (p)+2-2 p}{(p-\log p-1)^{2}} .
\end{aligned}
$$

Hence, 
$\sigma^{2}\left(X_{0}\right)=E\left[X_{0}^{2}\right]-\left(E\left[X_{0}\right]\right)^{2}=a^{2} \frac{\log ^{2}(p)+2 \log (p)+2-2 p}{(p-\log p-1)^{2}}-a^{2}=a^{2} \frac{1+2 p \log p-p^{2}}{(p-\log p-1)^{2}}$

and

$u_{\mathcal{D}}(p, a)=\sigma\left(X_{0}\right)=a \frac{\left(1+2 p \log p-p^{2}\right)^{0.5}}{p-\log p-1}$,

and (A.18) and (A.19) follow from (A.11).

\section{APPENDIX B: Unimodal Class}

To avoid any ambiguity, the explicit definition of the unimodal class used in this article is given here. First, unimodality is defined.

Definition: Unimodality. A univariate distribution is unimodal if there exists a number $a$, called the mode or vertex, such that the cumulative probability distribution function (CDF) is convex on $(-\infty, a)$ and concave on $(a, \infty)$.

This definition covers the intuitive definition of unimodality which is based on the PDF. This implies that with an absolutely continuous CDF, unimodality of a distribution about $a$ indicates the existence of a density function that is non-decreasing on $(-\infty, a)$ and non-increasing on $(a, \infty)$, or that it has a single peak; see Fig. B.1. Further reference is made to [28]. A unimodal distribution is illustrated in the figure below.
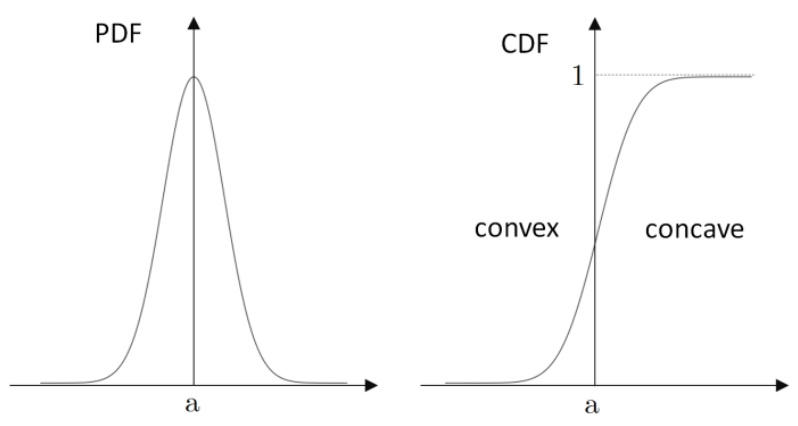

Fig. B.1. A univariate unimodal distribution.

Now, the unimodal class can be defined.

Definition: Unimodal Class. The unimodal class $\mathcal{P}\left(E X, \sigma(X)^{2}\right)$ is the set of all univariate distributions with the mode equal to the mean $E X$ and the variance $\sigma(X)^{2}$, as well as the condition on unimodality (defined above).

Gauss inequality [29] is a well-known subset of the (double-sided) Chebyshev inequality for the unimodal class. Gauss inequality suggests an improvement of the probability bound proposed by Chebyshev by a considerable factor of 4/9, being the merit of the additional unimodality constraint. The original Gauss inequality is a double-sided inequality, whereas a one-sided version of it is required as related to the notion of exceedance probability discussed in this article. Therefore, the work by [13] is used to evaluate the upper bound of exceedance probabilities for the class of unimodal distributions. Such a one-sided bound is numerically calculated through solving a so-called semi-definite program (SDP), being an advanced optimization problem with matrix inequalities.

It is worth mentioning that the unimodal class does not fully cover the log-concave CDF class. This means that one might find distributions that reside in the log-concave CDF classes but are not unimodal. However, despite this potential overlap, the bound derived based on the log-concave CDF class is covered by the unimodal bound in most practical applications where small exceedance probabilities are in question. This is due to the generally much more restricted constraint imposed by the log-concavity condition, and the often-smaller size of the distributional set. This can be understood from Fig. 3, where the curves for these classes do not intersect for values of $\gamma$ above 2.1 (approximately)i.e., the tail of the distribution.

\section{Acknowledgement}


The first and third authors wish to acknowledge support from the Research Council of Norway through the Centre for Research-based Innovation, SAMCoT and the support from all SAMCoT partners. In addition, all authors would like to thank Hongtao Li and Dr. Torodd S. Nord at NTNU for providing the long-term ice time series. The authors are also grateful to the anonymous referees for their valuable comments and suggestions. 


\section{References}

[1] Calafiore GC, Ghaoui LE. On Distributionally Robust Chance-Constrained Linear Programs. J Optim Theory Appl. 2006;130:122.

[2] Moller B, Beer M. Engineering computation under uncertainty - Capabilities of non-traditional models. Computers \& Structures. 2008;86:1024-41.

[3] Beer M, Ferson S, Kreinovich V. Imprecise probabilities in engineering analyses. Mechanical Systems and Signal Processing. 2013;37:4-29.

[4] Savage IR. Probability inequalities of the Tchebyscheff type. Journal of Research of the National Bureau of Standards. 1961;65B:211-22.

[5] Isii K. On a method for generalizations of tchebycheff's inequality. Ann Inst Stat Math. 1959;10:65-88.

[6] Isii K. Inequalities of the types of chebyshev and cramér-rao and mathematical programming. Ann Inst Stat Math. 1964;16:277-93.

[7] Marshall AW, Olkin I. Multivariate chebyshev inequalities. The Annals of Mathematical Statistics. 1960:1001-14.

[8] Bertsimas D, Sethuraman J. Moment Problems and Semidefinite Optimization. In: Wolkowicz H, Saigal R, Vandenberghe L, editors. Handbook of Semidefinite Programming: Springer US; 2000. p. 469-509.

[9] Lasserre JB. Bounds on measures satisfying moment conditions. Annals of Applied Probability. 2002:1114-37.

[10] Bertsimas D, Popescu I. Optimal Inequalities in Probability Theory: A Convex Optimization Approach. SIAM Journal on Optimization. 2005;15:780-804.

[11] Popescu I. A semidefinite programming approach to optimal-moment bounds for convex classes of distributions. Mathematics of Operations Research. 2005;30:632-57.

[12] Vandenberghe L, Boyd S, Comanor K. Generalized Chebyshev Bounds via Semidefinite Programming. SIAM Review. 2007;49:52-64.

[13] Van Parys BG, Goulart P, Kuhn D. Generalized Gauss inequalities via semidefinite programming. Math Program, Ser B. 2015:132 .

[14] Grechuk B, Molyboha A, Zabarankin M. Chebyshev inequalities with law-invariant deviation measures. Probability in the Engineering and Informational Sciences. 2010;24:145-70.

[15] An MY. Logconcavity versus Logconvexity: A Complete Characterization. Journal of Economic Theory. 1998;80:350-69.

[16] Bagnoli M, Bergstrom T. Log-Concave Probability and Its Applications. Economic Theory. 2005;26:445-69.

[17] Walther G. Inference and Modeling with Log-concave Distributions. Statistical Science. 2009;24:319-27.

[18] Cantelli FP. Sui confini della probabilita. Atti Congr In ternaz Matemat. 1928;6:47-59.

[19] Faridafshin F. Alternative Methodologies for Structural Design under Uncertainty: With a Focus on the Design of Arctic Offshore Structures Protected by Ice Management, Doctoral Thesis: Norwegian University of Science and Technology; 2017.

[20] Madsen HO, Krenk S, Lind NC. Methods of Structural Safety: Dover Publications; 2006.

[21] Sengupta D, Nanda AK. Log-concave and concave distributions in reliability. Naval Research Logistics (NRL). 1999;46:419-33.

[22] Li H, Bjerkås M, Høyland KV, Nord TS. Panel loads and weather conditions at Norströmsgrund lighthouse 2000-2003. Proceedings of the 23rd IAHR International Symposium on Ice. Ann Arbor, Michigan USA2016.

[23] Athreya KB, Lahiri SN. Measure theory and probability theory: Springer Science \& Business Media; 2006.

[24] Föllmer H, Schied A. Stochastic finance, volume 27 of de Gruyter Studies in Mathematics. Walter de Gruyter \& Co., Berlin, extended edition; 2004.

[25] Rockafellar RT, Uryasev SP, Zabarankin M. Deviation measures in risk analysis and optimization. University of Florida, Department of Industrial \& Systems Engineering Working Paper. 2002.

[26] Rockafellar RT, Uryasev S, Zabarankin M. Generalized deviations in risk analysis. Finance and Stochastics. 2006;10:51-74.

[27] Hanoch G, Levy H. The efficiency analysis of choices involving risk. The Review of Economic Studies. 1969;36:335-46.

[28] Dharmadhikari SW, Joag-dev K. Unimodality, Convexity, and Applications: Academic Press; 1988.

[29] Gauss CF. Theoria combinationis observationum erroribus minimis obnoxiae, pars prior. Commentationes Societatis Regiae Scientiarum. 1821;33:321-7. 